\title{
Intelligent bus temperature measurement system based on Zigbee networks
}

\author{
Gang $\mathrm{Ma}^{1, \mathrm{a}}$, WenGong Jiang ${ }^{2, \mathrm{~b}}$ \\ ${ }^{1}$ Uonone Group Co,.Ltd., Yangzhong 212211, China; \\ ${ }^{2}$ Jiangsu Wetown Busway CO,LTD., Yangzhong 212211, China; \\ awtwenbo@163.com, bjwg@wetown.cc
}

\begin{abstract}
Keywords: High-voltage switch cabinet; Wireless temperature measurement method; Zigbee networks; Data validation.
\end{abstract}

\begin{abstract}
In order to avoid overheating due to bus power supply system that will cause major accidents, we need to monitor the temperature of high-voltage switch cabinet in the narrow space. This paper designs a wireless intelligent bus temperature measurement system based on Zigbee networks. The application of wireless temperature measurement method in this system brings about the high voltage isolation, direct connection temperature acquisition, Zigbee networks technology with data validation and the solution to the insulation interference problem due to high voltage and magnetic field. Data processing terminals map and store data statistics, and provide a convenient for us to inquiry and research the switch cabinet usage. This temperature measurement system makes real-time monitoring on high-voltage switch cabinet's bus condition, it allows staff to discover the fault symptom in time, facilitate our power outage and maintenance plan. This system improves work efficiency, reduces the failure rate of the equipment and improves the power system's reliability and security.
\end{abstract}

\section{Introduction}

With the development of electric power system, power system's nationwide networking has become inevitable. The demands of relay protection that ensures its safe and stable operation will increase, too. A lot of traditional relay protection technology already can't meet the requirements of power system. Bus is the foundation of the whole power system, much current runs through it. Because of the connection material, installation, contact area, pressure, or aging, bus resistance will increase, which will directly cause the temperature increase[1]. If the temperature rise can't be discovered by staff in time, serious accidents will happen. Improving the security and stability of the bus has great significance to the whole power system.

High-voltage switch cabinet is an important equipment to power plants and transformer substations. Accidents caused by overheating of bus connection happen every year. As a result, we must monitor the bus temperature of the high-voltage switch cabinet, in order to avoid the accident caused by bus overheating. While the voltage in the high-voltage switch cabinet is too high to be manual measured. What's worse is that the space in the high-voltage switch cabinet is narrow, which makes difficult for wiring. Therefore, researching a kind of wireless bus temperature measuring system is imperative.

\section{Relevant Theories}

Bus temperature monitoring mainly involves temperature measuring device and wireless communication technology. With the rapid development of science and technology, the research on temperature measuring device and wireless communication technology constantly deepened. For the moment, high-voltage switch cabinet temperature measurement mainly concludes Stencil paper test temperature method, Infrared temperature measurement method, Optical fiber temperature measurement and Wireless temperature measurement method. Stencil test temperature method 
determines temperature through the stencil melting and shedding situation of the temperature measuring part. This method has low real-time and accuracy. Infrared temperature measurement method is widely used in medium and small substations, this method get temperature value through dealing with infrared radiation energy of the measured points instead of contact temperature measurement, it is expensive poor effective and unable to measure the temperature of the contact device in a closed state. Optical fiber measurement is an advanced technology of temperature measurement, can test temperature in real time, but its high cost, difficulty in wiring, installation and remove influences its promotion in the power industry. This paper uses Wireless temperature measurement method, temperature measuring device is installed in high pressure area, the collected temperature information is sent to the intelligent terminal through the wireless module. This method has the advantages of high accuracy, good real-time performance, low cost and low power consumption[2-4].

Another important part of the bus temperature monitoring is the wireless communication technology. Commonly used wireless communication technologies include infrared communication technology, bluetooth technology, WiFi technology and Zigbee technology[5-9]. We choose Zigbee technology in the aspects of cost, power consumption, reliability and convenience. Zigbee technology is a two-way wireless communication technology with low complexity, low cost, low power consumption, safety and reliablity, easy networking, wide application, etc.

In this paper, a kind of intelligent bus temperature measurement system based on Zigbee networks is established. The system uses single chip microcomputer control technology, digital signal processing technology and Zigbee communication technology. It can display the temperature of the bus in real time, process the temperature data and perform a series of operations when the threshold is exceeded.

\section{Overall Design of System}

According to the function demand of bus temperature measurement, this system includes four parts: temperature measurement module, intelligent terminal, digital process terminal and remote intelligent terminal. The temperature measurement module measures temperature data and sends it to the intelligent terminal by Zigbee networks. Terminal displays data and alarms. Intelligent terminal transmit temperature data to digital process terminal through the serial bus. Remote intelligent terminal accesses to the digital process terminal through Internet. Overall structure is shown in Fig. 1.

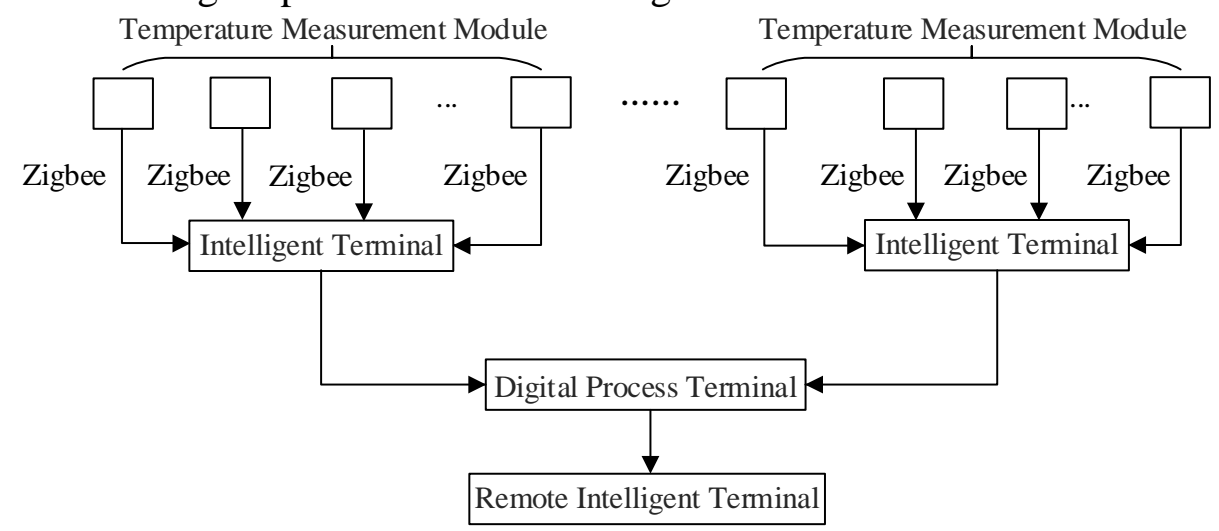

Fig. 1 Overall system structure diagram of temperature measurement device

Temperature measurement module consists of power module, temperature sensor and wireless data transceiver, mainly aims at temperature data sampling. It is shown in Fig. 2. The power module provides electricity for temperature sensor and wireless data transceiver by the battery[10]. Temperature measurement module is installed on the bus and its temperature sensor contacts with the bus through the aluminum box. Temperature sensor transmits temperature data to the wireless data transceiver through data line after it measures the temperature. Wireless data transceiver transmits the collected data to the higher level intelligent terminal through Zigbee networks. 


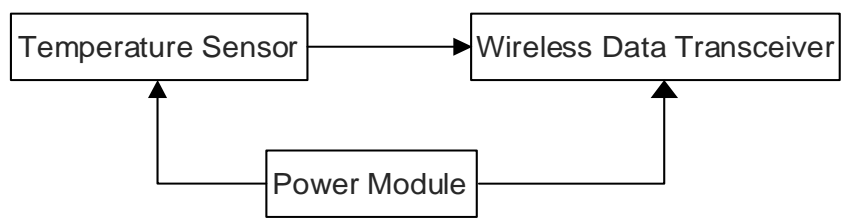

Fig. 2 The structure diagram of temperature measurement module

Intelligent terminal consists of wireless data transceiver, microprocessor, independent type button, light and alarm. It has five main functions, such as module selection, data examination, temperature limit alarm, indicator light display and communication. Its structure diagram is shown in Fig. 2. Its power module takes electricity from the bus to supply power to the microprocessor, the wireless data transceiver and the LED.

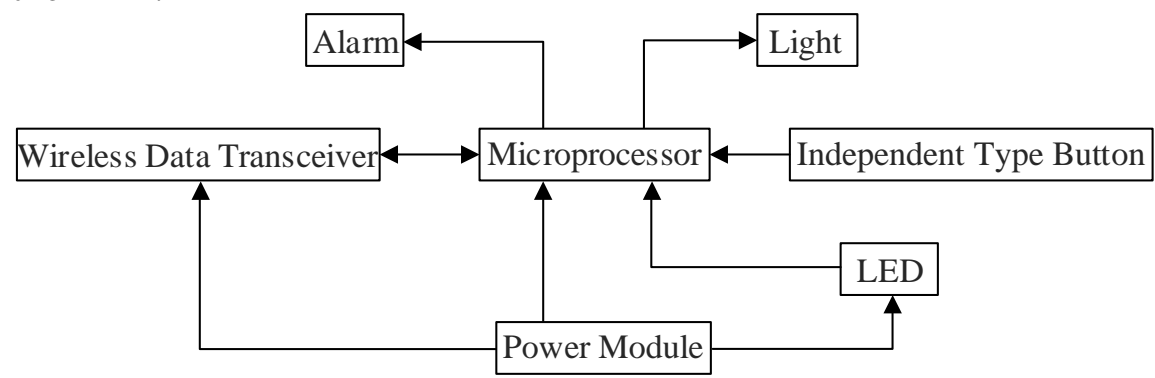

Fig. 3 The design diagram of the intelligent terminal

The conmunication between intelligent terminal and temperature measurement module is shown in Fig. 4. The intelligent terminal contacts with temperature measurement module through Zigbee networks in broadcasting mode. Microprocessor sends data to the wireless data transceiver and sends broadcasting orders to all the temperature measurement modules, these broadcasting orders contain the addresses of temperature measurement modules. The temperature measurement module which correspond with the address in the order sends messages to the intelligent terminal. The mode of the intelligent terminal changes into receiver mode after the intelligent terminal sends the message, and the wireless data transceiver receives message and transmits it to the microprocessor. Microprocessor supervises on the independent type button to make sure its display mode. It also examines and displays the collected data and judges whether it exceeds the alarm limit. Data process terminal aims to collect data, draw the corresponding histogram and storage them, in order to query and research the historical data.

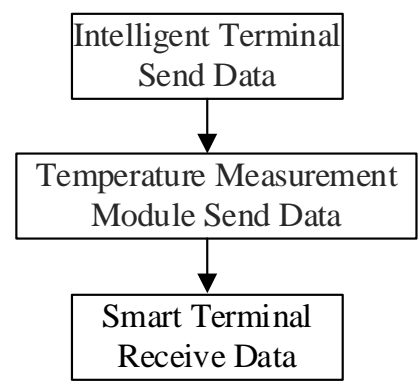

Fig. 4 The flow chart of data collection

Remote intelligent terminal mainly use internet to communicate with substation or power station in order to monitor real-time data remotely. Smart terminal is equipped with a liquid crystal display which can show the temperature of each bus on site.

The bus intelligent temperature measurement system can work in LAN mode as a complete temperature measurement system when the digital process terminal can't communicate with a remote intelligent terminal through Internet, This system can measure the real-time temperature and display, but can’t communicate with the substation or power station. The system structure is shown in Fig. 5. 


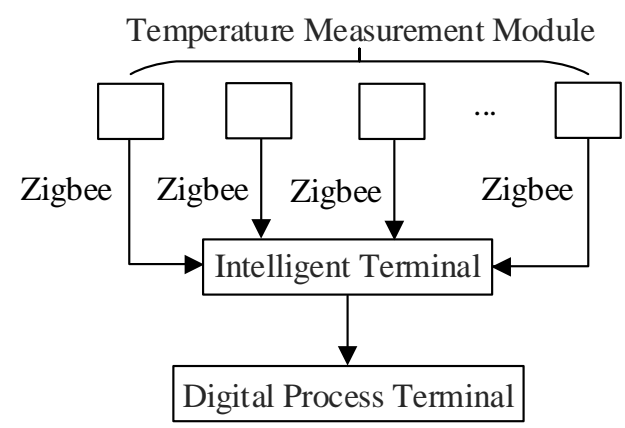

Fig. 5 LAN system configuration diagram of temperature measuring device

\section{System Algorithm Design}

The intelligent terminal is the core component of the bus intelligent temperature measurement system. It is not only responsible for receiving and checking the monitored data, but also for sending the data. Intelligent terminal has two modes: the rotation mode and the selection mode. It also has a separate button to select the display mode.

If the display mode is the rotation mode, the initial address will be determined by the microprocessor. Then, data in the corresponding position is collected, checked to ensure its accuracy. The accurate data is shown on the LED and determined whether it has reached the threshold value we set. If it is more than the threshold vaule, the system will start the alarm and the corresponding indicator lights and wait for manual reset. If it does not reach the threshold, the address will increase and then determine whether the address overflows, if not, the temperature data corresponding to the new address will be collected, otherwise, the system resets the initial address.

If the display mode is the selection mode, the destination address should be entered in the stand-alone keyboard and the microprocessor reads the address to complete the collection temperature data of the target address. The data must be checked, displayed and determined whether it exceeds the threshold. If it exceeds, the system starts the alarm and waits for a reset signal, otherwise, the system waits the reset signal directly. The algorithm is shown in Fig. 6.

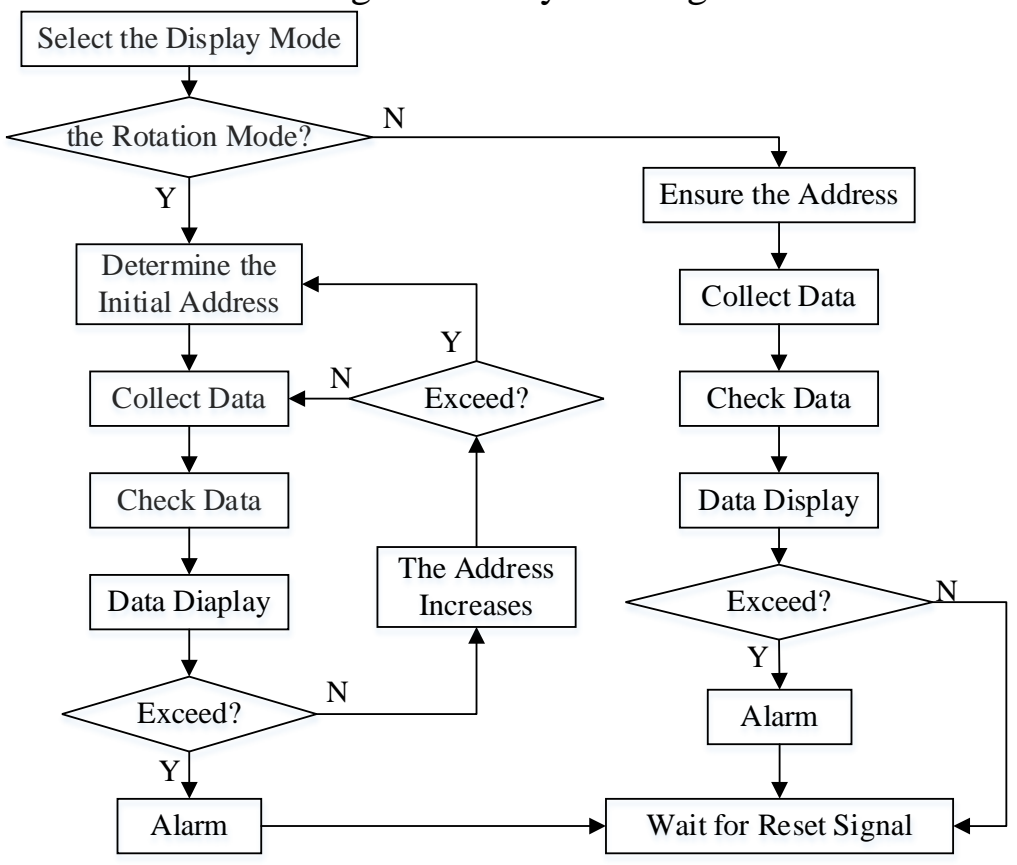

Fig. 6 Intelligent terminal system flow chart

The electromagnetic interference will affect the accuracy of the data transmission, so we need to check the data we received. First, the system provides the initial temperature value $\alpha$, and the wireless data transceiver receives the data. Then, the system gets an estimated value $\beta$ according to the former K-1 data of temperature measurement modules and Kalman filter calculation, and the 
absolute error of estimated value is $\gamma=|a-\beta|$, the absolute error maximum is set to be $1^{\circ} \mathrm{C}$. In the end, if $0 \leqslant \gamma \leqslant 1$, the data is correct, and the data validation completes; If $\gamma>1$, the data is wrong, the system saves $\alpha$ and continues to calculate with the estimated vaule $\beta$ until the data is correct. Specific calibration process is shown in Fig. 7. After the calibration, the data is show on the built-in LED by the software in microprocessor.

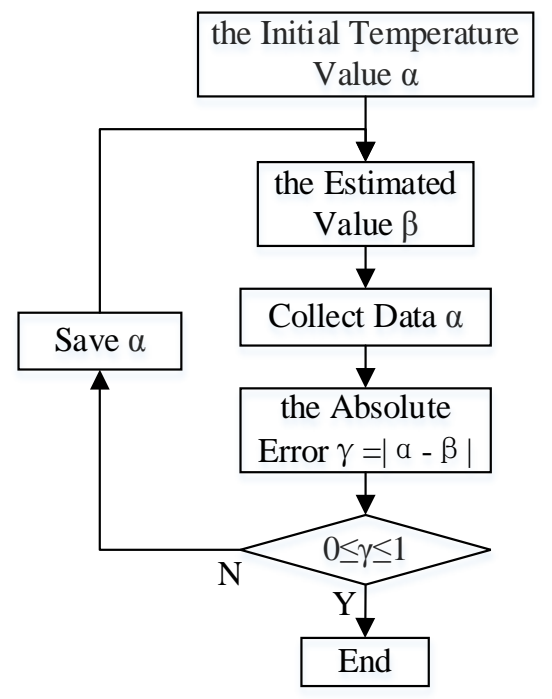

Fig. 7 Intelligent terminal data validation flow chart

\section{System Application}

This paper establishes an intelligent bus temperature measurement system which can be used to detect the rise of temperature of the bus in high-voltage switch cabinet, in order to prevent all kinds of malignant accidents caused by bus overheating. System's temperature measurement module is installed directly on the bus. Its sensor contacts directly with the bus. The temperature measurement module transfers the temperature data to intelligent terminal through Zigbee networks. The intelligent terminals are equipped with LED screen and independent type button, for real-time displaying bus temperature rise conveniently. Intelligent terminal can also connect with digital process terminal by USB to upload real-time data. Digital process terminal collects real-time data, maps and stores data statistics. Remote intelligent terminal monitors digital process terminal through Internet and shows bus temperature codition. Installation structure is shown in Fig. 8.

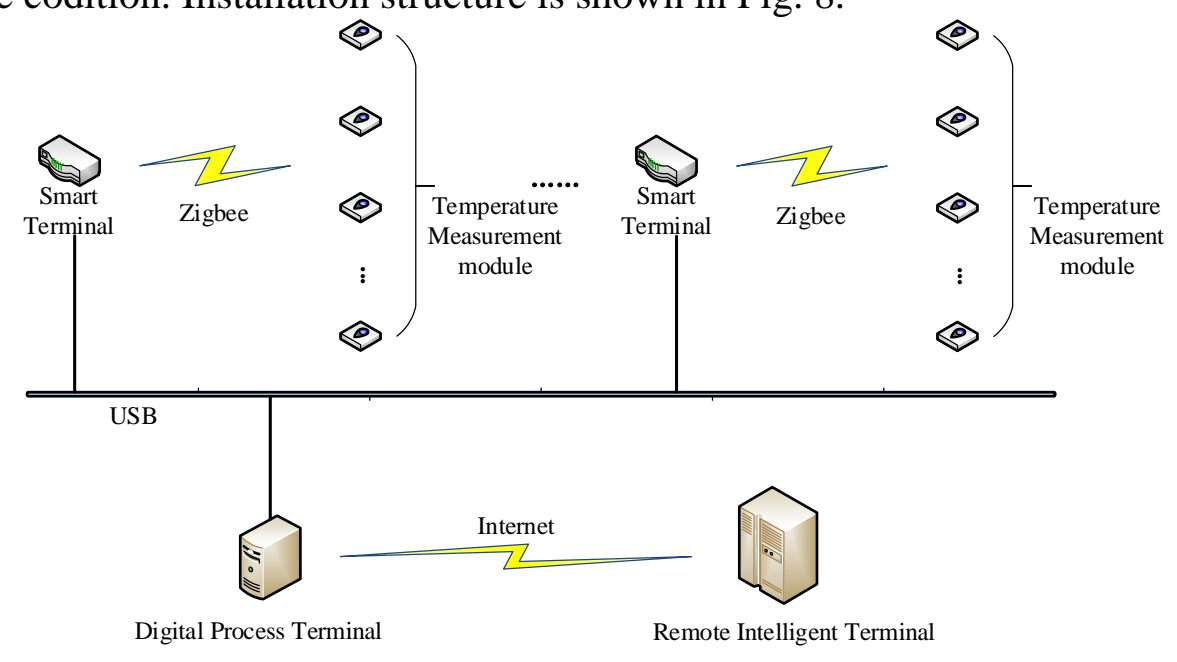

Fig. 8 Wireless smart bus temperature-measuring system installation structure

On-site staff can examine equipment bus's temperature rise by intelligent terminal operation. Operators can check the distant temperature of high voltage switchgear equipment through the remote intelligent terminal, so we can know the running status of equipment. It allows us to discover the fault symptom in time, facilitate our power outage and maintenance plan, improve work 
efficiency and reduce the failure rate of the equipment. The wireless intelligent bus temperature measuring system can work normally in open fields, office buildings, residential and commercial, making full effect on bus temperature monitoring, in order to improve the reliability and security.

\section{Summary}

This paper designs a wireless intelligent bus temperature measurement system based on Zigbee networks. The application of wireless temperature measurement method in this system brings about the high voltage isolation, direct connection temperature acquisition, Zigbee networks technology with data validation and the solution to the insulation interference problem due to high voltage and magnetic field. Data process terminals map and store data statistics, and provide a convenient for us to inquiry and research the switch cabinet usage. The temperature measurement system can monitor high voltage switchgear bus temperature condition online to ensure its safe operation.

\section{References}

[1]. ZHANG Shui-xiang, GAN Hui. Temperature monitoring system for HV switches based on fiber-optic temperature sensor. High Voltage Apparatus. Vol. 44 (2008) No.6, p. 513-515.

[2]. WANG Yang-guang, YIN Xiang-gen, YOU Da-hai. Application of Wireless Sensor Networks in Smart Grid. Power System Technology. Vol. 34 (2010) No.5, p. 7-11.

[3]. JIN Zhendong, XU Zhen, JIN Feng, YUAN Changjin. Summary And Analysis of Temperature Measurement Model for Domestic HV Electrified Equipment. Electrical Equipment. Vol. 8 (2007) No.12, p. 58-61.

[4]. HAO Xuelei, CHEN Peng, WANG Kunpeng, LV Kun. Application of Cable Temperature on-Line Monitoring System Based on Distributed Temperature Sensing. Telecom Power Technology. Vol. 30 (2013) No.2, p. 59-60.

[5]. HUANG Wei-hong, XIE Zhang-hong, CHEN Xiang-wei, JIANG Xiu-bo. Design and Application of Wireless Temperature Monitoring System for Switchgear Assemblies Based on Zigbee Technology. High Voltage Apparatus. Vol. 49 (2013) No.181, p. 125-130.

[6]. YAN De-li, HU Li-qiang, GAO Zhan-feng. Design and realization of wireless temperature monitoring system based on Zigbee. Logistics Engineering and Management. Vol. 31 (2009) No.2, p. 64-65.

[7]. ZHU Wen, YUAN Cheng, ZHANG Su. Design and Implementation for Substation Wireless Temperature Monitoring System. Water Resources and Power. Vol. 31 (2013) No.6, p. 207-210.

[8]. XU Bo, YANG Fan. The Research of Wireless Temperature Measurement System for the High-voltage Switchgear. Electrical Measurement and Instrumentation. Vol. 49 (2012) No.562, p. 91-95.

[9]. LIANG Hu-hui, ZHANG Feng, CHANG Chong, ZHAO Tao. ZigBee-based substation monitoring and alarming system. Power System Protection and Control. Vol. 38 (2010) No.12, p. 121-124

[10]. TIAN Yi, HUANG Xinbo. On-line monitoring system of temperature inside HV switchgear cabinet. High Voltage Apparatus. Vol. 46 (2010) No.3, p. 64-67. 